\title{
Explaining Function with Anatomy: Language Lateralization and Corpus Callosum Size
}

\author{
Goulven Josse, Mohamed L. Seghier, Ferath Kherif, and Cathy J. Price \\ Wellcome Trust Centre for Neuroimaging, UCL, London WC1N 3BG, United Kingdom
}

The anatomy of the corpus callosum (CC) has been advocated as a potential marker for functional lateralization because its size is supposedly proportional to the number of fibers connecting the hemispheres. Previous morphometric studies of this relationship have compared CC size in groups of subjects who are more or less likely to show differences in their lateralization (e.g., left vs right handers). The findings, however, have been inconsistent, and to our knowledge, no previous study has directly compared CC size with lateralization assessed by functional imaging data. We therefore combined anatomical measurements of CC size with left versus right hemisphere language activation in 74 normal subjects. After controlling for perceptual and motor output effects, as well as for global white-matter volume, handedness, gender and age, we found that subjects who had a larger CC showed more left lateralization for language in posterior temporal and inferior frontal regions. Examination of these effects revealed that, as $\mathrm{CC}$ size increased, stronger lateralization resulted from more left hemisphere activation in both regions as well as reduced right hemisphere activation in the posterior temporal region. Our observations provide the first clear evidence in normal subjects that the midsagittal surface area of the CC contributes to the degree to which language is functionally lateralized. We discuss the complex interhemispheric processes that might underlie this effect.

Key words: corpus callosum; language; lateralization; fMRI; anatomy; variability

\section{Introduction}

Lateralization of language function is one of the well-established characteristics of the brain (Broca, 1865; Dax, 1865; Hécaen et al., 1981), yet the reasons behind lateralization are unknown. Previous studies have highlighted the contribution of the underlying structural anatomy and proposed that structural asymmetries and interhemispheric connections could reflect or determine functional lateralization (Geschwind and Levitsky, 1968; Geschwind and Galaburda, 1985; Witelson, 1989). However, very few studies have combined anatomy and function to test this hypothesis (for review, see Josse and Tzourio-Mazoyer, 2004). Here, we consider the relationship between the macroscopic anatomy of the corpus callosum (CC) and functional lateralization for language.

The CC is the main anatomical link between the left and right hemispheres, consequently it plays an important functional role in interhemispheric interactions (Gazzaniga, 2000). The relationship between CC size and functional lateralization has been supported by several lines of previous evidence. First, fiber density has been found to be the same in small and large CCs, sug-

Received Sept. 9, 2008; revised 0ct. 6, 2008; accepted Nov. 12, 2008

This work was supported by the Wellcome Trust and the James S. MacDonnell Foundation (conducted as part of the Brain Network Recovery Group initiative). We also thank our three radiographers (Amanda Brennan, Janice Glensman, and David Bradbury) and the following people for recruiting subjects and helping with fMRI and behavioral data collection: Clare Shakeshaft, Laura Stewart, Tom Schofield, Alice Grogan, Hwee Ling Lee, Anneke Haddad, Fulden Babur, Caroline Ellis, and Odette Megnin. Guillaume "Magic G" Flandin was very helpful with data analysis. Finally, we thank the two reviewers for their helpful comments.

Correspondence should be addressed to Goulven Josse, Wellcome Trust Centre for Neuroimaging, UCL, 12 Queen Square, London WC1N 3BG, UK. E-mail: gjosse@fil.ion.ucl.ac.uk.

D01:10.1523/JNEUROSCI.4383-08.2008

Copyright $\odot 2008$ Society for Neuroscience $\quad 0270-6474 / 08 / 2814132-08 \$ 15.00 / 0$ gesting that when the midsaggital surface area of the CC is larger, there are more interhemispheric connections (Aboitiz et al., 1992). Second, postmortem and structural imaging studies have reported that the midsaggital surface area of the CC varies with hand preference and this has been related to the well known differences in the degree of language lateralization in left and right handers (Witelson, 1989). However, the results from this set of studies have been inconsistent (Steinmetz et al., 1992; Jäncke et al., 1997; Luders et al., 2003), perhaps because of small sample sizes. In addition, CC size has been shown to correlate positively with right hemisphere dominance for speech as assessed with the Wada test but this finding could be related to postlesional plasticity and thus be specific to the type of patients investigated (O'Kusky et al., 1988). CC size has also been negatively related to dominant right-ear performance as assessed with dichotic listening, a finding interpreted as reflecting more inhibition of the nondominant hemisphere on the dominant hemisphere in subjects with a larger CC (Clarke and Zaidel, 1994).

In contrast to previous studies, the current study used a direct measure of functional cerebral lateralization to provide a quantifiable relationship between CC size (midsaggital surface area) and the degree of language lateralization in the healthy brain. To do this we combined structural and functional MRI measurements from a sample of 74 normal participants. We looked at whether anatomical measurements of CC size could explain left versus right language activation using a whole-brain voxel by voxel analysis. Having established the brain regions where activation becomes more or less lateralized with CC size, we examined activation in each hemisphere independently to establish whether lateralization differences were driven by increased activation in the dominant left hemisphere or de- 


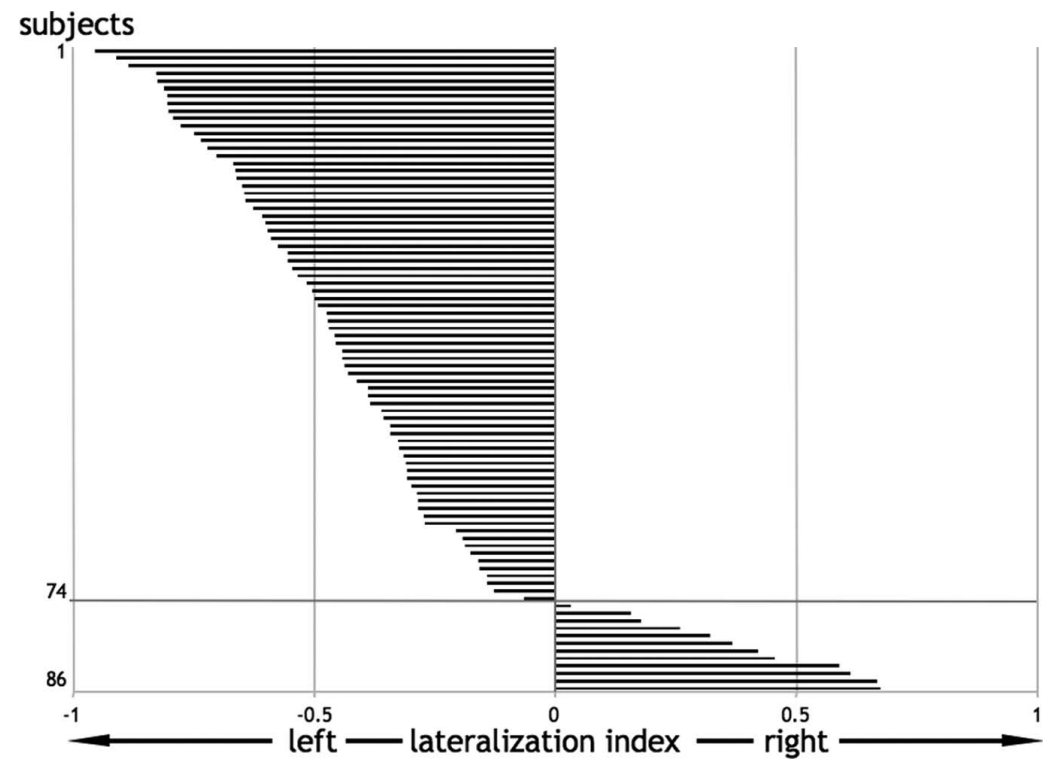

Figure 1. Hemispheric lateralization index for each subject. 0 f 86 subjects, 74 were selected who showed left or no lateralization (Nagata et al., 2001) for all language tasks taken as a whole and contrasted to their specific baselines. Negative values indicate more activated voxels (independent of threshold) on the left than on the right and vice versa.

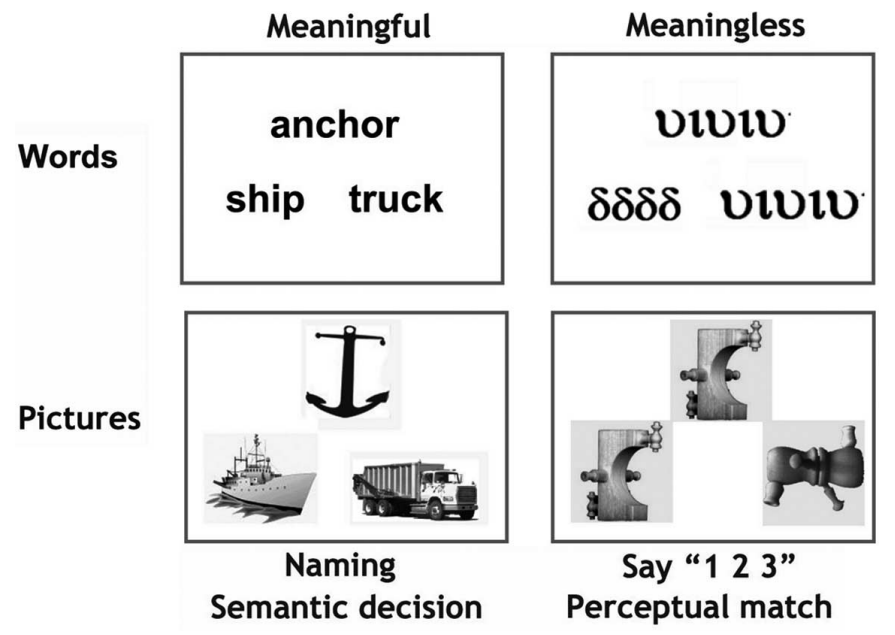

Figure 2. Examples of the stimuli used in the functional MRI paradigm. For the sake of simplicity and to show that items were matched over tasks, the words correspond to the pictures in this particular example. However, to avoid subjects from focusing on semantic relations during naming, items within triads were rearranged for the naming condition. See Materials and Methods for details.

creased activation in the nondominant right hemisphere. This approach allows us to associate functional lateralization to the underlying brain structure (Geschwind and Galaburda, 1985) and to make inferences about interhemispheric interactions during language processing.

\section{Materials and Methods \\ Subjects}

This study was approved by the local Ethics Committee, and all subjects gave written informed consent to take part in the study. All subjects were native english speakers and were free of any psychiatric or neurological abnormalities. From a total of 86 subjects with anatomical and functional scans, we selected 74 who showed a negative or null lateralization index, suggesting left or no lateralization for language processing in our paradigm of interest (Fig. 1) (see below for details of paradigm and the assessment of laterality). The remaining 12 subjects, with lateralization toward the right hemisphere, were excluded because our focus was on the relationship between CC size and the degree of left lateralization, not on the determinants of left versus right lateralization (interestingly, however, among the 12 nonselected right-lateralized subjects, only one subject was a self-described right hander) (Hécaen et al., 1981). It is also possible that CC size may have opposite effects in those with rightlateralized language compared with those with left-lateralized language. In our final sample of 74 subjects, there were equal numbers of males and females. Thirty-two subjects described themselves as right handed and the remaining 42 described themselves as either ambidextrous or left handed. These self-descriptions were concordant with the Edinburgh questionnaire (Oldfield, 1971) (supplemental Table A; supplemental Fig. A, available at www. jneurosci.org as supplemental material).

\section{Experimental design}

The language activation paradigm used a $2 \times 2$ factorial design which resulted in 4 activation conditions (Fig. 2). The first factor manipulated "stimulus" which could be either pictures of objects or their written names. The second factor manipulated "task," which could be either naming or semantic decision. Each of the four activation conditions had a corresponding sensorimotor baseline condition. For picture stimuli, the baseline involved seeing pictures of nonobjects and for written words, the baseline involved seeing unfamiliar symbols. This resulted in a total of eight conditions: four activation tasks and four baseline tasks. In all trials for all conditions, three stimuli were simultaneously presented as a "triad," with one stimulus above and two stimuli below. The task instructions were as follows.

Picture naming. Subjects were instructed to name aloud all three pictures in the triad, starting with the top stimulus, then the lower left stimulus and finally the lower right stimulus. Emphasis was put on saying the words with as little movement of the jaws as possible. A microphone was used to record the vocal responses and subsequently distinguish between correct and incorrect trials.

Picture naming baseline. To control for sensory and motor processing, subjects articulated "one, two, three" aloud while looking at the three pictures of unfamiliar nonobjects. Responses were recorded as in the naming task.

Reading words and reading baseline. The instructions were the same as for picture naming and its baseline, i.e., read all three object names and say "one, two, three" in response to the unfamiliar symbols.

Semantic decisions on pictures of objects. Subjects were required to make a finger press response to indicate whether the stimulus above (e.g., anchor) was more closely related in meaning to the stimulus on the lower left (e.g., ship) or the lower right (e.g., truck). Reaction times and accuracy were recorded. The selected response (left or right side of screen) was indicated by the spatial position of two fingers on the same hand. Thus, the right middle finger indicated the stimulus on the right whereas the index finger of the same hand indicated the stimulus on the left.

Semantic baseline. To control for sensory, motor and decision making processing, the baseline task required participants to decide whether the unfamiliar stimulus above looked identical to the stimulus on the lower left or lower right. Subjects responded using the same finger press responses as in the semantic conditions.

Semantic decisions on written names and perceptual decisions on unfa- 
miliar symbols. The instructions and recordings were identical to those described for pictures above.

Each subject participated in four scanning runs lasting $\sim 6$ min each, with two runs involving the four articulation tasks and two runs involving the four matching (finger press) tasks. Within each run, stimuli were blocked with four triads per block. Each run included four blocks (16 triads) of pictures, four blocks of words, as well as two blocks (eight triads) of nonobjects and two blocks of symbols. Each block was preceded by 3.6 s of instructions (e.g., "Name," "Read," "Say 1,2,3"), and each triad then remained on the screen for $4 \mathrm{~s}$ followed by $180 \mathrm{~ms}$ of fixation, adding up to $16.7 \mathrm{~s}$ for each condition. In addition, we interspersed the task blocks with four blocks of fixation each lasting $14.4 \mathrm{~s}$. The order of words and pictures was counterbalanced within run. In addition, the order of task was counterbalanced across runs (either run 1: articulation, run 2: matching, run 3: matching, and run 4: articulation; or run 1: matching, run 2: articulation, run 3: articulation, and run 4: matching).

\section{Stimuli}

All stimuli were derived from a set of 192 familiar objects with three to six letter names: 33 had three-letter names (e.g., "cat," "bus," "hat,"...), 65 had four-letter names ("ship," "bell," "frog," "hand,"...), 58 had fiveletter names ("teeth," "camel," "snake,"...), and 36 had six-letter names ("spider," "dagger," "button,"...). A pilot study with eight subjects ensured intersubject agreement on all picture names. The 192 objects were first divided into two different sets of 96 items which we will refer to as set $A$ and set $B$. One group of selected subjects was presented with set A as written words for reading aloud, set $B$ as pictures for object naming, set $B$ for semantic decisions on words, and set A for semantic decisions on pictures. The other group was presented with set B as written words for reading aloud, set $\mathrm{A}$ as pictures for object naming, set $\mathrm{A}$ for semantic decisions on words, and set $\mathrm{B}$ for semantic decisions on pictures. Thus, no word or picture was repeated over the experiment, although each object concept occurred twice (once as a word and once as a picture). Words and pictures were counterbalanced within and between runs.

In the naming/reading triads, we minimized the semantic relationship between stimuli, e.g. "lemon" (above), "cow" (lower left), "pipe" (lower right). In the semantic triads, there was a strong semantic relationship between two items in the triad but not the third item, e.g., "anchor" is more semantically related to "ship" than "truck." We did not include triads where the semantic decision could be made on the basis of perceptual attributes or verbal associations (e.g., "cat" and "dog," "knife" and "fork," "sock" and "shoe"). A pilot study with eight subjects ensured intersubject agreement on the expected semantic association.

Stimulus presentation was via a video projector, a front-projection screen and a system of mirrors fastened to a head coil. Words were presented in lower case Arial and occupied 4.9 degrees (width) and 1.2 degrees (height) of the visual field. Each picture was scaled to take $7.3^{\star} 8.5$ degrees of the visual field.

\section{Data acquisition}

A Siemens 1.5T Sonata scanner was used to acquire both anatomical and functional images from all subjects. Anatomical T1-weighted images were acquired using a three-dimensional modified driven equilibrium Fourier transform sequence and 176 sagittal partitions with an image matrix of $256 \times 224$ and a final resolution of $1 \mathrm{~mm}^{3}$ [repetition time (TR), 12.24/echo time, 3.56/inversion time, $530 \mathrm{~ms}$ ].

Functional $\mathrm{T} 2{ }^{\star}$-weighted echoplanar images with blood oxygenation level-dependent contrast comprised 40 axial slices of $2 \mathrm{~mm}$ thickness with $1 \mathrm{~mm}$ slice interval and $3 \times 3 \mathrm{~mm}$ in-plane resolution. Onehundred and three volumes were acquired per session, leading to a total of 412 volume images across the four sessions. Effective TR was 3.6 s/volume. TR and stimulus onset asynchrony did not match, allowing for distributed sampling of slice acquisition across the experiment (Veltman et al., 2002). To avoid Nyquist ghost artifacts, a generalized reconstruction algorithm was used for data processing. After reconstruction, the first four volumes of each session were discarded to allow for T1 equilibration effects.

\section{Data analysis}

Image processing and statistical analyses were conducted using statistical parametric mapping (SPM5: Wellcome Trust Center for Neuroimaging, London, UK; http//www.fil.ion.ucl.ac.uk) running under Matlab 7 (Mathworks).

\section{Anatomical MRI data}

Preprocessing of the structural images first involved manual anteriorposterior commissure (AC-PC) realignment. Images were then segmented and spatially normalized using the unified segmentation/normalization approach implemented in SPM5 (Ashburner and Friston, 2005). The surface of the CC was drawn midsagitally on each subject's unnormalized segmented white-matter image. This was actually done in five centered contiguous slices, the surface areas of which were then averaged. AC-PC alignment allowed for comparable measurements across subjects (Witelson, 1989). Segmentation made ROI delineation easier. In addition to looking at the effect of the whole CC size on language lateralization, we looked for effects specific to subdivisions of the $\mathrm{CC}$ along the anteroposterior axis. Such subdivisions are based on different connectivity patterns (posterior CC connecting posterior cortical areas, anterior CC connecting anterior areas) the relative size of which varies between subjects, suggesting different parts of the CC could explain functional lateralization in different ways (Witelson, 1989). However, these subdivisions have been delineated based mainly on anatomical studies in nonhuman primates (Witelson, 1989). We therefore used a more recent parcellation scheme of the CC based on human data: five different CC subdivisions were identified along the anteroposterior axis according to Hofer's diffusion tensor imaging (DTI) scheme (Hofer and Frahm, 2006). The surface areas of these CC subdivisions could be retrieved via a simple voxel count. We refer to their surface areas as "Hofer I," "II," "III," "IV," and "V," the total midsaggital surface area being referred to as "CC" in tables. The different areas were drawn separately, for each subject, on the basis of relative proportions [Hofer and Frahm (2006), their Fig. 3]. The average total CC midsagittal surface area per subject was $601 \mathrm{~mm}^{2}(\mathrm{SD}=92)$, which is concordant with data reported in the literature (Jäncke et al., 1997; Luders et al., 2003).

To control for global white-matter volume, which could explain part of CC variance, we also measured the total white-matter volume of the two hemispheres. To exclude the cerebellum, the midbrain and the CC, we defined a mask of the hemispheres on the SPM template that was then applied to each subject's normalized white-matter image. This normalized image had voxel values that were modulated by the relative volume before and after spatial normalization; therefore, white-matter volume in native space could be reliably estimated by simply adding voxel values and multiplying by voxel size (Goldszal et al., 1998; Ashburner and Friston, 2001). The mean total white-matter volume of both hemispheres was $418 \mathrm{ml}(\mathrm{SD}=49)$.

\section{Functional MRI data}

Preprocessing. Each subject's functional volumes were realigned and unwarped (Andersson et al., 2001), adjusting for residual motion-related signal changes. Scans from the different subjects were then spatially normalized. This normalization was based on the unified segmentation/ normalization of the structural image after it had been coregistered to the functional images. The template used was a symmetrical version of the default structural template from the Montreal Neurological Institute implemented in SPM5. This symmetrical template was created by simply copying, flipping along the $x$-axis and averaging the original and the mirror versions of the template. The resulting normalization parameters were then applied to the subject's functional images thereby rendering them symmetrical. This was relevant to the statistical analyses where we directly compared left and right hemisphere activation by including both flipped and unflipped contrast images (see below) (Jäncke et al., 2002; Baciu et al., 2005). The normalized functional images were then spatially smoothed with a $6 \mathrm{~mm}$ full-width half-maximum isotropic Gaussian kernel to compensate for residual variability after spatial normalization and to permit application of Gaussian random-field theory for corrected statistical inference (Friston et al., 1995).

First level analysis. At the first level, data were analyzed in a subject- 
specific manner. Correct responses for each of the eight conditions, the instructions as well as the errors were modeled separately, with eventrelated delta functions for each trial, convolved with a canonical hemodynamic response function. Condition effects were estimated according to the general linear model. To exclude low frequency confounds, the data were high-pass filtered using a set of discrete cosine basis functions with a cutoff period of $128 \mathrm{~s}$. The contrasts of interest at the first level were each of the four activation conditions relative to their specific baseline condition, i.e., picture naming versus saying "1 23 " to nonobjects; reading words versus saying " 123 " to unfamiliar letters; semantic decisions on pictures versus perceptual match of nonobjects; semantic decisions on words versus perceptual match of unfamiliar letters.

These four contrast images, for each subject, were then copied, and the copies were flipped along the $x$-axis. The resulting original (unflipped) symmetrical contrast images and their flipped versions could then be entered into second level ANOVAs to compare left and right hemisphere activation and permit inferences across subjects.

Lateralization indices. Before proceeding with the second level voxel based analyses, we first excluded 12 subjects because their laterality index at the global level was positive, suggesting right lateralization. Different methodological issues may influence the computation of a laterality in$\operatorname{dex}$ (Seghier, 2008); therefore, we used here a relatively robust procedure that allows the laterality index to be assessed over tasks and thresholds. Practically, the lateralization index we used was computed for each subject using the average of the four unflipped contrast images [for a similar rationale, see Ramsey et al. (2001)] and calculating the number of activated voxels in each hemisphere independently of the statistical threshold (Nagata et al., 2001). All but one of the 12 excluded subjects with a positive (right $>$ left) lateralization index were self-described non-right handers (Hécaen et al., 1981). The remaining 74 subjects (whose data were included in the voxel by voxel statistical analyses described below) showed a continuum of language lateralization ranging from no lateralization to strong left lateralization (Fig. 1).

Main effect of language lateralization at the voxel level. Before exploring the effect of CC size on language lateralization, we identified the main effect of lateralization independent of CC size. This provided regions of interest that were useful for determining whether the effect of CC size colocalized or not with the main effect of lateralization. The analysis included the eight different contrast images for each of the 74 selected subjects, i.e., the flipped and unflipped versions of the contrasts for each of the four tasks relative to its baseline. The comparison of unflipped versus flipped images identified left or right hemisphere voxels showing significant differences between hemispheric activation. The statistical threshold was $p<0.05$ after family-wise error (FWE) correction for multiple comparisons across the whole brain.

\section{Combining anatomical with functional MRI data}

The effect of CC size on language lateralization was estimated in another second level ANOVA that was identical to the one used to look at the main effect of lateralization, except it included two covariates corresponding to total CC size and white-matter size, respectively. The effect of CC size on language lateralization corresponded to the difference in the regression of CC size on unflipped versus flipped images. In each statistical contrast, activation was always relative to the task-specific baseline. Thus, the effect of task $=$ (naming and reading $>$ saying $1,2,3$ to nonobjects and unfamiliar symbols) versus (semantic decisions on objects and written names $>$ perceptual decisions on nonobjects and unfamiliar symbols) and the effect of stimulus $=$ (pictures of objects $>$ nonobjects) versus (written names $>$ unfamiliar symbols). This resulted in a whole-brain statistical parametric map from which we identified the voxels where the significance of the effect of CC size survived FNE correction $(p<0.05)$ for multiple comparisons either across the whole brain or across areas showing a main effect of lateralization. To clarify the issue of whether any effect of CC size could be related to handedness, gender or age, we performed the same ANOVA adding these three variables as regressors along with CC size and white-matter volume.

Having identified the voxels where language lateralization (unflipped vs flipped images) depended on CC size, we then conducted a series of post hoc analyses.
Table 1. The main effect of language lateralization

\begin{tabular}{llllll}
\hline & \multicolumn{5}{l}{$Z$ scores } \\
\cline { 3 - 4 } Anatomical location & Coordinates $(x, y, z)$ & $\mathrm{L}>\mathrm{R}$ & $\mathrm{L}$ only & $\mathrm{R}>\mathrm{L}$ & $\mathrm{R}$ only \\
\hline Occipitotemporal sulcus & $-46,-56,-16$ & $\operatorname{Inf}$. & 13.4 & & \\
Pars opercularis & $-54,14,18$ & $\operatorname{Inf}$ & 8.2 & & \\
Paracingulate sulcus & $-6,10,52$ & 6.7 & 12.7 & & \\
Supramarginal gyrus & $-56,-48,24$ & 5.2 & 1.4 & & \\
Cerebellum & $32,-66,-26$ & & & 6.7 & 8.9
\end{tabular}

Areas where the comparison of unflipped versus flipped images was significant at $p<0.05$ (after FWE correction for multiple comparisons across the whole brain). Coordinates are specific to our symmetrical template. They are only given for comparison between regions in the present study. L, Left hemisphere; R, right hemisphere; Inf., inferior.

To identify whether lateralization differences with CC size reflected activation increases in the left dominant hemisphere or activation decreases in the right nondominant hemisphere, we report the effect of CC size on left and right homolog regions using the original unflipped images. These effects are reported at an uncorrected threshold because they are limited to preidentified voxels.

To identify whether the effect of total CC size was driven by specific subdivisions ("Hofer I," "II," "III," "IV," and "V"), we reran the analyses several times replacing the total CC regressor with the corresponding effect for one Hofer-specified CC subdivision in addition to the sum of the remaining measurements for the other subdivisions, i.e., in ANOVA \#2 "Hofer I" and "CC minus Hofer I" were used as regressors, in ANOVA \#3 "Hofer II" and "CC minus Hofer II," etc.

To relate our anatomofunctional findings to the existing anatomical literature, we also explored the relationship between CC size and factors such as handedness, gender, age and white matter. These analyses were again restricted to the 74 selected subjects, to be comparable with the main anatomofunctional analyses of lateralization.

\section{Results}

\section{The main effect of language lateralization}

The main effect of left $>$ right language lateralization (direct comparison of unflipped vs flipped images) was most significant in the occipitotemporal sulcus, inferior frontal gyrus, paracingulate sulcus and supramarginal gyrus (Table 1; Fig. 3). These effects extended to a large portion of the superior temporal sulcus. The only right $>$ left effect was observed in the cerebellum.

\section{The effect of CC size on language lateralization}

The central result of this study is that CC midsaggital surface area correlated positively with left language lateralization in two regions (Table 2; Fig. 4; supplemental Fig. B, available at www. jneurosci.org as supplemental material, supplemental plots). In the whole-brain search $(p<0.05$ FWE corrected for multiple comparisons across the whole brain), CC size predicted the degree of left lateralization in a posterior middle temporal region localized at the level of or in front of the anterior occipital sulcus (depending on which individual normalized brain was used for localization). We will refer to this area as the "temporal region." It was not significantly lateralized on average across subjects, although it was on the border of areas showing a main effect of lateralization. When the search was limited to areas showing a significant main effect of lateralization, a positive effect of CC size on left $>$ right hemisphere activation was localized in the posterior part of the inferior frontal sulcus at the level of the precentral sulcus. We will refer to this area as the "inferior frontal sulcus" or simply "frontal region." This effect was highly significant when correcting for multiple comparisons within the main effect of lateralization and also approached significance when correcting for multiple comparisons across the whole brain ( $p=0.068$ FWE corrected). In both the temporal and frontal regions, activation was more left lateralized for all four tasks in those with a larger CC despite controlling for sensory input, motor output and global 
white-matter volume. Although the $Z$ scores were higher for naming than semantic decisions, there was no significant interaction $(p>0.05)$ between task and the effect of CC size on language lateralization.

Whole white-matter volume did not have an effect on lateralization, even when removing CC size from the model.

Finally, these results were not affected when handedness, gender and age were included as regressors along with CC size and white-matter volume, only making the $p$ value associated with the effect of CC size in the frontal region slightly smaller and now significant when correcting for comparisons across the whole brain $(p<$ 0.05 FWE corrected). However, as expected, handedness had an additional effect on language lateralization such that right handers showed stronger left lateralization for language in a region of the inferior frontal sulcus close to the region where an effect of CC size was found (supplemental Fig. C, available at www.jneurosci.org as supplemental material). This was due to a positive effect of handedness on the left (more activation in right handers) and to a negative effect of handedness on the right (more activation in left handers).

\section{More left hemisphere activation or less right hemisphere activation?}

In the temporal region, the effect of CC size on lateralization over all tasks was partly explained by increased left hemisphere activation $(Z=3.3)$ and partly explained by reduced right hemisphere activation during the naming condition $(Z=3.0)$. In contrast, in the frontal region, the effect of CC size was primarily driven by a left hemisphere increase $(Z=5.2)$ rather than by a right hemisphere decrease (Table 2).

\section{The contribution of different CC subdivisions}

We did not find any evidence that the effect of CC on language lateralization was greater for one subregion (i.e., Hofer I, II, III, IV, or V) relative to the others. This null result is explained by the observation that the size of each subregion strongly correlated with all others with the exception of Hofer IV with Hofer I and V (supplemental Table B.b, available at www.jneurosci.org as supplemental material). Therefore it was not possible for us to tease apart the effect of one subregion relative to another. The correlations between subdivisions are not surprising because the subdivisions were defined as proportions in the $y$-axis. Therefore, if the total CC increased, all subdivisions increase in the same proportion in the $y$-axis. To tease apart the effect of one CC subregion relative to another on language lateralization therefore relies on intersubject variability in the size of each subregion in the $z$-axis.

\section{The relationship between CC size and other potential lateralization factors}

Total CC surface area was positively correlated with white-matter volume $(p<0.001)$ (supplemental Table B.a; supplemental Fig. $\mathrm{D}$, available at www.jneurosci.org as supplemental material) and this relationship was also observed in each of the five subdivisions (Hofer I, II, III, IV or V). In Hofer II, CC size was related to handedness and age, being larger in non-right handed and younger subjects (supplemental Figs. E, F; supplemental Table
Table 2. The effect of CC size (Z score) on language lateralization (left > right)

\begin{tabular}{|c|c|c|c|c|c|c|}
\hline & \multicolumn{3}{|c|}{$\begin{array}{l}\text { Posterior temporal region } \\
(-/+44,-60,2)\end{array}$} & \multicolumn{3}{|c|}{$\begin{array}{l}\text { Inferior frontal region } \\
(-1+44,10,20)\end{array}$} \\
\hline & Left $>$ right & Left & Right & Left $>$ right & Left & Right \\
\hline All language & $5.2^{*}$ & 3.3 & -3.0 & $4.7^{*}$ & $5.2^{*}$ & NS \\
\hline All naming & $5.0^{*}$ & 2.7 & -3.1 & 3.4 & 2.9 & -1.79 \\
\hline All semantic & 2.7 & 2.0 & NS & 3.6 & 4.7 & NS \\
\hline
\end{tabular}

Values in parentheses are the symmetrical $x, y$, and $z$ coordinates. In the temporal and frontal regions with a significant effect of $\mathrm{CC}$ size on unflipped versus flipped images, we also report the effect of $\mathrm{CC}$ size at the same left hemisphere coordinates in the unflipped images only (i.e., left hemisphere) or the flipped images only (i.e., right hemisphere). ${ }^{*} p<0.05$ familywise error corrected for multiple comparisons. NS, Not significant at $p<0.05$, uncorrected.

B.a, available at www.jneurosci.org as supplemental material). The size of Hofer II was also smaller in non-right handed males and older females (supplemental Figs. E, F, available at www. jneurosci.org as supplemental material). However, these supplemental results should be qualified by the fact that the study was not designed to look at gender effects and groups of males and females were not matched for handedness (supplemental Fig. A, available at www.jneurosci.org as supplemental material).

\section{Discussion}

This study shows the first direct evidence that the midsaggital surface area of the CC partially predicts functional lateralization for language. There have been several studies of the macroanatomy of the CC (Witelson, 1989; Jäncke et al., 1997; Luders et al., 2003) or language lateralization in normal subjects (Mazoyer et al., 1993; Binder et al., 1996; Springer et al., 1999; TzourioMazoyer et al., 2002; Seghier et al., 2004), but no studies combining both anatomical and functional data in normal subjects to test whether CC size is actually related to lateralization. Using structural and functional MRI in a large group of 74 subjects and an analysis allowing for high-resolution voxel-wise comparison of activation in the left versus right hemisphere, we conclusively show that left lateralization for language increased with CC size.

Based on the role of the CC in connecting the hemispheres (Gazzaniga, 2000), as well as evidence that CC size reflects the degree of interhemispheric connections (Aboitiz et al., 1992), several studies have looked at CC size as a potential marker of functional lateralization (O'Kusky et al., 1988; Witelson, 1989; Clarke and Zaidel, 1994; Jäncke et al., 1997; Luders et al., 2003). However, the evidence has not been convincing because it was 


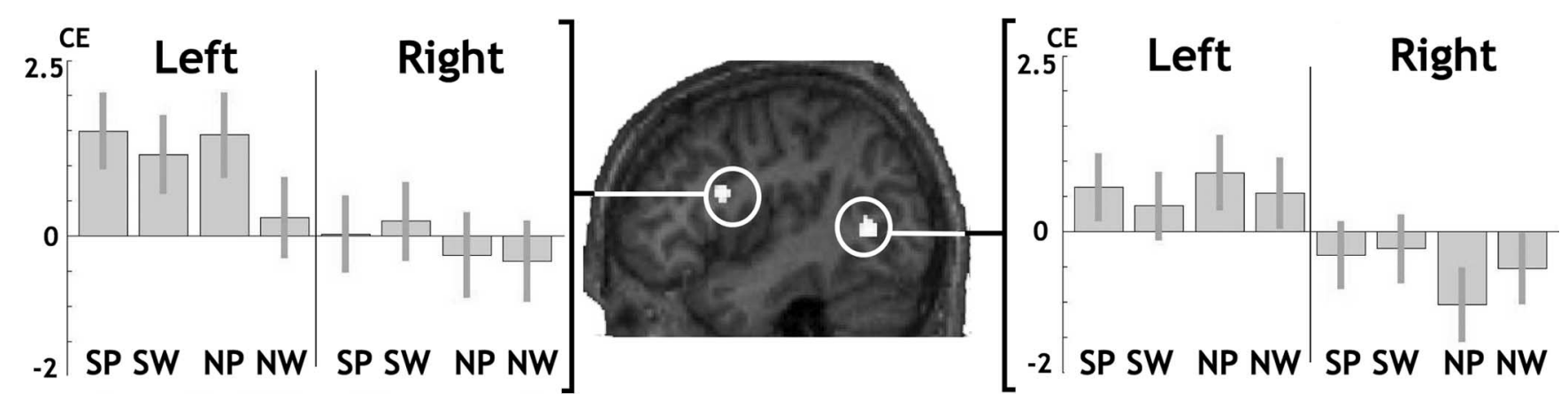

Figure 4. The effects of CC size on language lateralization. The sagittal section shown in the middle is from one individual brain normalized onto our symmetric template. The two regions showing a positive effect of $C($ size on left $>$ right language lateralization are overlaid in white ( $p=0.001$ uncorrected for multiple comparisons/minimum cluster size $=30$ voxels, each cluster containing a peak significant at $p<0.05 \mathrm{FWE}$ corrected for multiple comparisons). Panels to the left and to the right give details of unilateral CC effects during each condition in each of these regions (bar graphs with $90 \%$ confidence intervals). CE, Contrast estimate; SP, semantic decisions on pictures; SW, semantic decisions on words; NP, naming pictures of objects; NW, naming written object name.

based on indirect measures such as the comparison of groups thought, but not shown, to have different functional lateralizations. Focusing on a particular set of language tasks and without any a priori knowledge of which cortical regions were functionally related to CC size, we were able to confirm and specify an anatomofunctional relation between CC size and language lateralization. Specifically, we show that subjects with a larger CC are more likely to show a stronger left lateralization for language.

The positive relationship that we observed between CC size and left lateralization contrasts with previous work suggesting a larger CC resulted in less marked language lateralization (Witelson, 1989). This suggestion was logically inferred from the observation that non-right handed subjects had a larger CC, while it was known that this population showed less language lateralization on average (Hécaen et al., 1981). Critically, however, these previous anatomical studies did not directly measure language lateralization. Our data suggests that both handedness and CC size are related to language lateralization, but in independent ways. The effect of CC size was not affected by whether the effect of handedness was factored out or not. This is despite the fact that, as expected, handedness also had an effect on lateralization, with stronger left lateralization for right handers [for review, see Josse and Tzourio-Mazoyer (2004)].

What could then explain the negative effect of right handedness observed on CC size if it is unrelated to language lateralization as we measured it? Interestingly, this handedness effect was significant on a part of the CC (Hofer II) which connects premotor and supplementary motor areas in humans (Hofer and Frahm, 2006). This is consistent with handedness being a measure of motor lateralization with left $>$ right brain asymmetry in the motor system being greater for right handers than non-right handers (Dassonville et al., 1997; Amunts et al., 2000; Solodkin et al., 2001; Hervé et al., 2006). However, the previously reported negative effect of right handedness on CC size (Witelson, 1989) was not observed on the subpart of the CC where we found a negative effect of right handedness (Hofer II) (Hofer and Frahm, 2006). This apparent inconsistency may relate to differences in the way the CC was partitioned. Our partitioning scheme was based on DTI data in humans whereas most other studies have used partitioning schemes based on interhemispheric connections in monkeys, rather than humans (Hofer and Frahm, 2006). The critical point for the current study is that the effects of handedness, gender and age on CC size did not explain the effect of CC size on language lateralization.

Why should a larger corpus callosum be associated with stronger lateralization for language? Differences in CC size reflect the extent to which the hemispheres are connected (Aboitiz et al., 1992), and therefore the answer to that question should lie in determining the variable interhemispheric processes that underlie the positive relationship between CC size and language lateralization. For that purpose, we looked at whether the difference in left versus right hemisphere activation was driven by left hemisphere activation increases or right hemisphere activation decreases. Importantly, different unilateral effects of CC size were found in the temporal region and in the frontal region. Lateralization was bilaterally driven in the temporal region (increased left hemisphere activation as well as decreased right hemisphere activation) but unilaterally driven in the frontal region (primarily driven by increased left hemisphere activation). This posterior/ anterior dichotomy in language lateralization is concordant with the idea that hemispheric specialization for language is not a uniform phenomenon and has complex task and region dependent characteristics (Clarke and Zaidel, 1994; Luders et al., 2003; Cohen and Dehaene, 2004; Josse and Tzourio-Mazoyer, 2004).

What can we further infer about interhemispheric interactions from the posterior/anterior dichotomy in the processes driving language lateralization? Left-right balance in the temporal region might reflect more transfer of information for language processing from right to left in subjects with larger CC. Alternatively, there may be more inhibition of redundant right hemisphere activation in subjects with a larger CC. As for the unilateral effect in the left inferior frontal sulcus, it might result from right to left interhemispheric information transfer from sites other than the right homolog. This is possible because (1) in addition to homolog connections, there are heterolog connections between the hemispheres (Marconi et al., 2003) and (2) our result shows an effect of the size of the whole CC. We therefore infer that, in the temporal region, subjects with a larger CC have more inhibition in the right hemisphere and/or more information transfer from the right to the left hemisphere. In the frontal region, in contrast, the absence of right inferior frontal activation decreases in those subjects with a large CC suggests that left inferior frontal activation in these subjects reflects more information transfer either from right hemisphere homolog or heterolog regions.

Importantly, the temporal and frontal regions where language lateralization increased with CC size were in the vicinity of the main effect of language lateralization across subjects. This reinforces the idea that CC size is indeed related to language lateralization and not just to the lateralization of more variable areas less consistently involved in language. However, this needs to be qualified by the fact that our paradigm was limited to single-word production and semantic decision. Further studies are therefore 
required to look at the relationship between CC size and other language processes, for example auditory and syntactic tasks. In addition, our analysis targeted a population whose language activation was either bilateral or left lateralized. More studies are therefore required to explore whether there is also a positive relationship between CC size and language lateralization in subjects whose language activations are right lateralized or whether CC size is one of the determinants of left versus right lateralization for language. Additionally, while midsagittal surface area of the CC presumably reflects the number of fibers connecting the hemispheres, it would be interesting to test how this measure and its relation to functional data compares with other measures of interhemispheric structural connectivity, such as fractional anisotropy of the CC (Putnam et al., 2008).

Finally, previous work has suggested that the evolution of brain lateralization was driven by increasing brain size because of increasing spatial and timing constraints (Ringo et al., 1994; Jäncke et al., 1997; Rilling and Insel, 1999; Gazzaniga, 2000). Here, intraspecies, whole white matter-volume did not have an effect on lateralization. Furthermore, this hypothesis is partly based on observations that, as brain size increases, relative CC size decreases. It is also based on the assumption that a smaller CC corresponds to more lateralization. Interestingly, our result goes in the opposite direction, showing that, when whole whitematter volume is controlled for, a smaller CC is associated with less lateralization. It is therefore possible that differences in CC size correspond to more or less lateralization, depending on the context, for example, task and species.

In conclusion, we have shown that the degree to which language activation is left lateralized is partially explained by the midsaggital surface area of the CC in normal healthy volunteers. Specifically, we show that subjects with a larger CC are more likely to show stronger left lateralization for single word naming and semantic decision tasks. In addition, we show that in one of the CC subdivisions (Hofer II), right handers have a smaller midsaggital surface area than non-right handers. This effect of handedness is consistent with previous studies (Witelson, 1989); however, the interpretation of the results is different. Previously, the negative effect of right handedness on CC size was taken to imply that language lateralization is stronger when CC size is smaller. In contrast, our study shows that the effect of CC on handedness does not correspond to the effect of CC on language lateralization measured by functional imaging. Our interpretation is therefore that language lateralization is stronger when CC size is larger. Finally, by exploring whether language lateralization was driven by left hemisphere activation increases and/or right hemisphere activation decreases, we propose that the effect of $\mathrm{CC}$ size on posterior temporal activation is partly driven by right hemisphere inhibition, whereas the effect of CC size on inferior frontal activation is driven by more information transfer to the left hemisphere from right hemisphere homolog or heterolog regions.

\section{References}

Aboitiz F, Scheibel AB, Fisher RS, Zaidel E (1992) Fiber composition of the human corpus callosum. Brain Res 598:143-153.

Amunts K, Jäncke L, Mohlberg H, Steinmetz H, Zilles K (2000) Interhemispheric asymmetry of the human motor cortex related to handedness and gender. Neuropsychologia 38:304-312.

Andersson JL, Hutton C, Ashburner J, Turner R, Friston K (2001) Modeling geometric deformations in EPI time series. Neuroimage 13:903-919.

Ashburner J, Friston KJ (2001) Why voxel-based morphometry should be used. Neuroimage 14:1238-1243.
Ashburner J, Friston KJ (2005) Unified segmentation. Neuroimage 26:839-851.

Baciu MV, Watson JM, Maccotta L, McDermott KB, Buckner RL, Gilliam FG, Ojemann JG (2005) Evaluating functional MRI procedures for assessing hemispheric language dominance in neurosurgical patients. Neuroradiology 47:835-844.

Binder JR, Swanson SJ, Hammeke TA, Morris GL, Mueller WM, Fischer M, Benbadis S, Frost JA, Rao SM, Haughton VM (1996) Determination of language dominance using functional MRI: a comparison with the Wada test. Neurology 46:978-984.

Broca P (1865) Sur le siege de la faculte du langage articule. Bull Soc Anthropol 6:377-399.

Clarke JM, Zaidel E (1994) Anatomical-behavioral relationships: corpus callosum morphometry and hemispheric specialization. Behav Brain Res 64:185-202.

Cohen L, Dehaene S (2004) Specialization within the ventral stream: the case for the visual word form area. Neuroimage 22:466-476.

Dassonville P, Zhu XH, Uurbil K, Kim SG, Ashe J (1997) Functional activation in motor cortex reflects the direction and the degree of handedness. Proc Natl Acad Sci U S A 94:14015-14018.

Dax M (1865) Lesions de la moitie gauche de l'encephale coincidant avec l'oubli des signes de la pensee (Lu au Congres Meridional tenu a Montpellier en 1836). Gaz Hebd Medecine Chir 2:259-260.

Friston KJ, Holmes AP, Poline JB, Grasby PJ, Williams SC, Frackowiak RS, Turner R (1995) Analysis of fMRI time-series revisited. Neuroimage 2:45-53.

Gazzaniga MS (2000) Cerebral specialization and interhemispheric communication: does the corpus callosum enable the human condition? Brain 123:1293-1326.

Geschwind N, Galaburda AM (1985) Cerebral lateralization. Biological mechanisms, associations, and pathology: I. A hypothesis and a program for research. Arch Neurol 42:428-459.

Geschwind N, Levitsky W (1968) Human brain: left-right asymmetries in temporal speech regions. Science 161:186-187.

Goldszal AF, Davatzikos C, Pham DL, Yan MX, Bryan RN, Resnick SM (1998) An image-processing system for qualitative and quantitative volumetric analysis of brain images. J Comput Assist Tomogr 22:827-837.

Hécaen H, De Agostini M, Monzon-Montes A (1981) Cerebral organization in left-handers. Brain Lang 12:261-284.

Hervé PY, Crivello F, Perchey G, Mazoyer B, Tzourio-Mazoyer N (2006) Handedness and cerebral anatomical asymmetries in young adult males. Neuroimage 29:1066-1079.

Hofer S, Frahm J (2006) Topography of the human corpus callosum revisited-comprehensive fiber tractography using diffusion tensor magnetic resonance imaging. Neuroimage 32:989-994.

Jäncke L, Staiger JF, Schlaug G, Huang Y, Steinmetz H (1997) The relationship between corpus callosum size and forebrain volume. Cereb Cortex $7: 48-56$.

Jäncke L, Wüstenberg T, Scheich H, Heinze HJ (2002) Phonetic perception and the temporal cortex. Neuroimage 15:733-746.

Josse G, Tzourio-Mazoyer N (2004) Hemispheric specialization for language. Brain Res Brain Res Rev 44:1-12.

Luders E, Rex DE, Narr KL, Woods RP, Jancke L, Thompson PM, Mazziotta JC, Toga AW (2003) Relationships between sulcal asymmetries and corpus callosum size: gender and handedness effects. Cereb Cortex 13:1084-1093.

Marconi B, Genovesio A, Giannetti S, Molinari M, Caminiti R (2003) Callosal connections of dorso-lateral premotor cortex. Eur J Neurosci 18:775-788.

Mazoyer BM, Tzourio-Mazoyer N, Frak V, Syrota A, Murayama N, Levrier O, Salamon G, Dehaene S, Cohen L, Mehler J (1993) The cortical representation of speech. J Cogn Neurosci 5:467-479.

Nagata SI, Uchimura K, Hirakawa W, Kuratsu JI (2001) Method for quantitatively evaluating the lateralization of linguistic function using functional MR imaging. Am J Neuroradiol 22:985-991.

O'Kusky J, Strauss E, Kosaka B, Wada J, Li D, Druhan M, Petrie J (1988) The corpus callosum is larger with right-hemisphere cerebral speech dominance. Ann Neurol 24:379-383.

Oldfield RC (1971) The assessment and analysis of handedness: the Edinburgh inventory. Neuropsychologia 9:97-113.

Putnam MC, Wig GS, Grafton ST, Kelley WM, Gazzaniga MS (2008) Structural organization of the corpus callosum predicts the extent and impact of cortical activity in the nondominant hemisphere. J Neurosci 28:2912-2918. 
Ramsey NF, Sommer IE, Rutten GJ, Kahn RS (2001) Combined analysis of language tasks in fMRI improves assessment of hemispheric dominance for language functions in individual subjects. Neuroimage 13:719-733.

Rilling JK, Insel TR (1999) Differential expansion of neural projection systems in primate brain evolution. Neuroreport 10:1453-1459.

Ringo JL, Doty RW, Demeter S, Simard PY (1994) Time is of the essence: a conjecture that hemispheric specialization arises from interhemispheric conduction delay. Cereb Cortex 4:331-343.

Seghier ML (2008) Laterality index in functional MRI: methodological issues. Magn Reson Imaging 26:594-601.

Seghier ML, Lazeyras F, Pegna AJ, Annoni JM, Zimine I, Mayer E, Michel CM, Khateb A (2004) Variability of fMRI activation during a phonological and semantic language task in healthy subjects. Hum Brain Mapp 23:140-155.

Solodkin A, Hlustik P, Noll DC, Small SL (2001) Lateralization of motor circuits and handedness during finger movements. Eur J Neurol 8:425-434.
Springer JA, Binder JR, Hammeke TA, Swanson SJ, Frost JA, Bellgowan PS, Brewer CC, Perry HM, Morris GL, Mueller WM (1999) Language dominance in neurologically normal and epilepsy subjects: a functional MRI study. Brain 122:2033-2046.

Steinmetz H, Jäncke L, Kleinschmidt A, Schlaug G, Volkmann J, Huang Y (1992) Sex but no hand difference in the isthmus of the corpus callosum. Neurology 42:749-752.

Tzourio-Mazoyer N, Landeau B, Papathanassiou D, Crivello F, Etard O, Delcroix N, Mazoyer B, Joliot M (2002) Automated anatomical labeling of activations in SPM using a macroscopic anatomical parcellation of the MNI MRI single-subject brain. Neuroimage 15:273-289.

Veltman DJ, Mechelli A, Friston KJ, Price CJ (2002) The importance of distributed sampling in blocked functional magnetic resonance imaging designs. Neuroimage 17:1203-1206.

Witelson SF (1989) Hand and sex differences in the isthmus and genu of the human corpus callosum. A postmortem morphological study. Brain 112: $799-835$. 